\title{
Power Systems Analysis Software for Fast Process Automation
}

\author{
Arnolda Rožanskiené ${ }^{1}$, Linas Markevičiuss ${ }^{2}$, Andronis Linas Markevičius ${ }^{2}$ \\ ${ }^{1}$ Department of Electric Power Systems, Kaunas University of Technology \\ Studentu str. 48, 51367 Kaunas, Lithuania \\ ${ }^{2}$ JSC "Protronika”, Šeiminiškių 5-82, Vilnius, Lithuania \\ arnoldarozanskiene@gmail.com; info@protronika.com
}

\section{Extended Abstract}

This paper presents innovative software solutions for Smart Power Management System [1]. Fast real time analysis of power systems stationary modes is achieved by finding the equivalent schemes for large and complex power networks, and fast analysis of transient processes is achieved by using digital filters method, and by modeling of power lines by the wave equations.

In power system's stationary mode analysis, one of the most time-consuming objects is load flow calculation. Duration of load flow computer analysis depends on power systems complexity level, which relates to a number of power lines and number of network diagram nodes. Increasing the level of complexity of the network diagram, the time of analysis is getting longer. The number of iterative cycles depends on the electrical system size and complexity [2]. Analysis time can be shortened by adequate equivalent network's diagram simplification including the reduction of number of single line diagram nodes. In order to evaluate the effectiveness of simplification, analysis was performed were simplified calculation time was compared with the time required to get the solution using any conventional calculation method. For the comparison was chosen the nodal voltage detection, with in advance found matrix of nodal impedances.

Calculation time depends on the number of cycles, and number of nodes in the network.

For better performance of network management could be useful the power systems reactive load flow control, with quick assessment of shunt reactors, capacitor banks or synchronous compensators [3]. Equipment selection is complicated due to unpredictable change of electricity production and electricity consumption. Selection of compensating equipment by limiting the voltages up to acceptable values or minimizing the power losses in the network, when electricity consumption is random and electricity generation is distributed, requires up to 1000 statistical test-calculations. Simplification of power network structure allows calculation time reduce down to a few seconds.

For automatic dispatch control, additionally to the stationary mode control must be carried out a rapid search of optimal and dynamically stable power system structures. Analysis of short circuit modes takes less time, as having founded the load flow distribution, iterative cycles are unnecessary. However, compare to the short circuits calculation, time for electromechanical transient process calculation increases as many times as many the discrete time steps have to be used for process monitoring. Studies have shown that calculation time does not exceed a few tens of milliseconds.

Fast electromagnetic processes are analyzed for identification of short circuit character, causes of the fault, and distance to the fault in power line, it is important after faults to determine the reliability indexes of possible network structures, when evaluated possible insulation damages by overvoltage. Voltage processes and their amplitudes after switching depends on many factors: initial phases at switching moment, residual charges, impedance at fault spot, etc.

Acceleration of calculations in electromagnetic transient processes software is achieved by separating the process mathematical modeling procedures from the parameters primary preparation part. Moreover, in procedures are maximally used various types of digital filters and matrix of differential equations. Studies have shown that the shortest computation time is achieved when modeling of overhead lines and cables is done by wave equations. Suitable wave attenuation and change of surge impedance are achieved by digital filters that are optimized in frequency spectrum. 


\section{References}

http://www.eurelectric.org/media/293202/winter_package_solutions-2016-030-0496-01-e.pdf

[2] R. Franke, H. Wiesmann, "Flexible modeling of electrical power systems - the Modelica PowerSystems library," Modelica Conference, Lund, Sweden, 2014.

[3] C. Bentsson, Z. Gajic, M. Khorami, "Dynamic Compensation of Reactive Power by Variable Shunt Reactors; Control Strategies and Algorithms," CIGRE, no. C1-303, 2012. 